\title{
Two Distinct Conformations of the Conserved RNA-rich Decoding Center of the Small Ribosomal Subunit Are Recognized by tRNAs and Release Factors
}

\author{
E.M. Youngman, L. Cochella, J.L. Brunelle, S. He, and R. Green \\ Howard Hughes Medical Institute, Department of Molecular Biology and Genetics, Johns Hopkins University \\ School of Medicine, Baltimore, Maryland 21205
}

\begin{abstract}
The mRNA is bound and poised in the decoding center of the small subunit of the ribosome where the genetic code is translated by the tRNAs, which recognize sense codons, and by the release factors, which recognize stop codons. Structural and biochemical studies have identified key universally conserved nucleotides, G530, A1492, and A1493, that are important for selection of cognate tRNA species during elongation. Here, we present evidence that these same universally conserved nucleotides are also important for interactions with the release factors, but must assume a very different structure during stopcodon recognition. These data provide mechanistic insight into how the decoding center of the ribosome has evolved to recognize distinct substrates with high fidelity, which in turn regulates the downstream chemical events of peptidyl transfer and peptide release.
\end{abstract}

The aminoacyl (A) site of the ribosome, spanning from the decoding center in the small subunit to the peptidyl transfer center in the large subunit, serves two related but distinct functions during translation. During each round of polypeptide elongation, a sense codon on the mRNA poised in the small subunit of the A site is recognized by its cognate aa-tRNA, bringing a new amino acid into the large subunit of the A site where peptidyl transfer occurs. During termination, the same site is bound by a release factor protein for recognition of a stop codon in the decoding site of the small subunit and catalysis of peptide release in the active site of the large subunit. Although peptidyl transfer and peptide release are two chemically related reactions that are catalyzed in the same active site on the ribosome, they are stimulated by the interactions that two very different "substrates" establish with the ribosome in a manner that ultimately depends on the codon sequence. It follows that these recognition events must take place with high fidelity, such that the two very different outcomes of peptidyl transfer and peptide release are carefully regulated.

Recent work has suggested that activation for peptidyl transfer is accomplished by conformational rearrangements in the active site promoted by interactions of the CCA end of the tRNA with the A loop of the peptidyl transferase center (PTC) (Schmeing et al. 2005; Brunelle et al. 2006). Although less is known about the structural and functional requirements for triggering peptide release in the PTC, a parsimonious view is that interactions with the A loop may similarly be critical for this regulated catalytic step (Caskey et al. 1971). It has also been established that in the context of an "activated" catalytic center, the universally conserved nucleotides in the PTC have no apparent catalytic role in promoting peptidyl transfer, although their identity is critical for peptide release (Youngman et al. 2004). Catalysis happens readily for the chemically facile aminolysis reaction (peptidyl transfer), whereas the inner core of conserved PTC nucleotides has a more substantial, as yet undefined, role in the chemically more challenging hydrolytic (peptide release) reaction. These data highlight how the ribosome has evolved an active site able to execute catalysis of two distinct reactions of varying chemical difficulty, and only in the context of bound substrate.

\section{THE DECODING CENTER AND THE ACCURACY OF TRANSLATION}

What is perhaps the more remarkable layer of complexity in regulation of this active site is that the two substrates that must bind to catalyze peptidyl transfer and peptide release are very different. Catalysis of peptide bond formation involves recognition of the codon presented in the A site of the decoding center by cognate aminoacyl tRNA, whereas peptide release involves recognition of stop codons in the same decoding center by protein release factors. Thus, like the large subunit active site, the decoding center of the small subunit must perform two related functions: recognition of RNA:RNA interactions during tRNA selection and recognition of RNA:protein interactions during release factor selection. Furthermore, recognition of the codons by these distinct components takes place with high accuracy that in large part dictates the overall fidelity of the translation of the genetic code (Cochella and Green 2005b). In vivo fidelity studies suggest that the overall accuracy of translation is extremely high, on the order of $10^{-3}$ to $10^{-4}$ for codon recognition by tRNAs and on the order of $10^{-5}$ for stopcodon recognition by release factors (Bouadloun et al. 1983; Parker and Holtz 1984; Jorgensen et al. 1993). It follows that the task of the ribosome, tRNAs, release factors, and relevant elongation factors is to specifically recognize cognate mRNA:tRNA and mRNA:release factor interactions while discriminating against near-cognate 
interactions that differ by a single-nucleotide substitution in the codon.

At the simplest level, there are clearly differences in the energy of the interaction between cognate and near-cognate partners, and these differences account for some of the observed fidelity. However, at least for the case of tRNA selection, it is clear that the overall energetic differences in these interactions cannot fully explain the observed fidelity of the process in vivo. For example, it has previously been pointed out that overall differences in the $\mathrm{G}: \mathrm{C}$ content of the codon:anticodon helix result in immediate discrepancies in binding energies which are not easily reconciled with the relatively uniform accuracy of protein synthesis (Ogle and Ramakrishnan 2005). Both GC- and AU-rich codon:anticodon helices are rather uniformly and accurately decoded by the protein synthesis apparatus. This apparent conundrum is rationalized by the view that it is the geometry of the codon:anticodon helix that is the critical feature recognized by the ribosome, allowing tRNA selection to occur with such high accuracy (Carter et al. 2000). This general view is well supported by kinetic studies which make the compelling argument that tRNA selection is kinetically driven by the acceleration of forward rate constants by cognate mRNA:tRNA interactions, at the expense of efficient utilization of differential off-rates of the cognate and nearcognate partners (Pape et al. 1999; Gromadski and Rodnina 2004a). More recent kinetic data have further argued that the ribosome assumes two distinct states in response to interactions in the decoding center-an "on" state and an "off" state-depending on whether correct or incorrect molecular partners are bound (Gromadski et al. 2006). These two states of the ribosome result in different downstream consequences: Cognate interactions accelerate forward rates (for GTPase activation and tRNA accommodation) and thus acceptance into the ribosome for subsequent events (peptidyl transfer), whereas nearcognate tRNAs do not accelerate these steps and thus are generally rejected from the ribosome. Although there is far less known about release factor recognition of stop versus near-stop codons, available data suggest that in this case as well, not all discrimination takes place at the level of differences in the energetics of the interaction between the mRNA and the release factor (Freistroffer et al. 2000). It seems possible that there will similarly be contributions to specificity made by the ribosome itself (e.g., the decoding center) in recognizing these precise interactions.

\section{MOLECULAR MECHANISM OF TRNA SELECTION}

Much is known about specific recognition of cognate tRNAs from high-resolution structures of the small ribosomal subunit. Early in the process of tRNA selection, docking of a cognate tRNA species with the codon of the mRNA triggers conformational rearrangements in universally conserved nucleotides in the decoding center. These changes include the rotation of a guanosine (G530) from a syn to an anti conformation by rotation around the glycosidic bond and the flipping of two adenosines (A1492 and A1493) from positions within helix 44 of $16 \mathrm{~S}$ rRNA to extrahelical positions (Ogle et al. 2001); we refer to this state as "flipped out." These seemingly unfavorable rearrangements are stabilized by the formation of A-minor interactions between nucleotides A1492, A1493, and G530 and the minor groove of the cognate codon:anticodon helix. These local interactions are thought in turn to favor the formation of a more global "closed" state of the ribosome observed in X-ray structures (Ogle et al. 2002). Formation of this closed state is thought to favor incorporation of the tRNA into the A site. Genetic variants that stabilize the closed state result in a decrease in the fidelity of protein synthesis (a ram, or ribosomal ambiguity, phenotype), whereas those that destabilize the closed state increase fidelity (known as restrictive) (reviewed in Ogle and Ramakrishnan 2005). Near-cognate codon:anticodon interactions, on the other hand, form irregular helices that do not stably interact with the flipped-out conserved adenosines and thus do not lead to ribosome closure and subsequent tRNA incorporation. In support of these models for tRNA selection, aminoglycoside antibiotics (such as paromomycin) known to induce miscoding during protein synthesis have been shown to bind in a pocket within helix 44 of the rRNA such that these two critical adenosines are specifically displaced from their uninduced position independent of the nature of the interaction in the decoding center (cognate vs. near-cognate) (Carter et al. 2000). Thus, paromomycin binding stabilizes the flipped-out conformation for these residues and triggers the associated downstream consequences for tRNA selection.

This summary of the kinetic and molecular determinants of high-fidelity tRNA selection by the ribosome yields an impressively detailed and consistent model for how this process takes place. Both functional and structural analyses of the related peptide release reaction lag substantially behind (Petry et al. 2005), although it is likely that these two processes are at least somewhat related. Although recognition of a specific codon by an RNA (a tRNA) and a protein factor (an RF) might depend on distinct properties of the molecular players, the downstream consequences of engagement of cognate species are clearly related: activation of the large ribosomal subunit catalytic center for the chemical reactions of aminolysis and hydrolysis. In the absence of detailed structural information about release factor engagement of the decoding center, we have taken a biochemical approach to decipher the molecular basis for the high fidelity of peptide release during protein synthesis. Here, we compare and contrast the relative contributions made by the universally conserved decoding center nucleotides to high-fidelity reading of sense codons by the tRNAs and of stop codons by the release factors.

\section{ROLE OF CONSERVED DECODING CENTER NUCLEOTIDES IN TRNA SELECTION AND PEPTIDE RELEASE}

Universally conserved nucleotides in the decoding center of the ribosome involved in specific, well-characterized molecular interactions with the codon:anticodon helix are thought to be central to the tRNA selection process (Ogle et al. 2001). We have used a site-directed 
mutagenesis approach to evaluate the contributions made by three key nucleotides, G530, A1492, and A1493, to decoding and peptide release. Mutations were incorporated in a plasmid-borne version of the $r r n B$ operon, and the variant ribosomes were expressed in the background of wild-type ribosomes (Powers and Noller 1990). All substitutions tested at these positions resulted in a dominant-lethal growth phenotype, requiring the use of an affinity purification system for the expression and subsequent isolation of variant ribosomes. Variant $30 \mathrm{~S}$ subunits were tagged with an MS2 RNA stem-loop inserted in the backbone of $16 \mathrm{~S}$ rRNA that allowed for affinity purification on a glutathione matrix via association with an MS2 coat protein fused to GST (Youngman and Green 2005).

Variant ribosomes were first evaluated in two assays - GTP hydrolysis and peptidyl transfer-that report on the critical rate-determining steps in tRNA selection of GTPase activation and accommodation, respectively (Gromadski and Rodnina 2004a). For both assays, ribosomal initiation complexes were formed by loading $70 \mathrm{~S}$ ribosome particles with a defined mRNA (with a Shine-Dalgarno sequence and an AUG start site followed by a UGG Trp codon) and an initiator tRNA (fMet-tRNA $\mathrm{f}_{\mathrm{fet}}^{\mathrm{Met}}$ ) using the requisite initiation factors (IFs1-3) (Cochella and Green 2005a). These ribosome complexes were then reacted with limiting amounts of a ternary complex of EF-Tu•GTP•Trp-tRNA ${ }^{\text {Trp }}$, and the rates of GTP hydrolysis and peptidyl transfer were measured. All three variants tested exhibited substantial decreases in the observed rates for these reactions (up to 40-fold for A1492G in each reaction) (Fig. 1). Given that these reactions were performed under nearly satu-
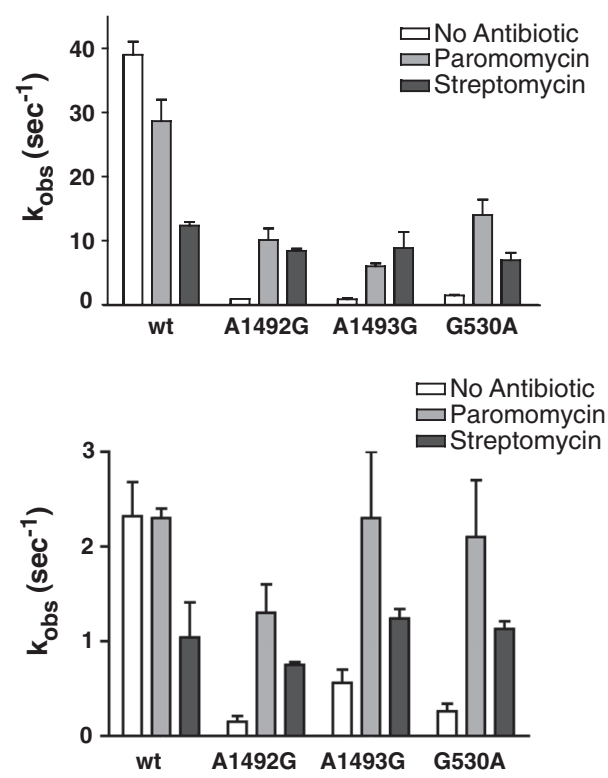

Figure 1. Aminoglycosides rescue the defects of decoding site mutant ribosomes in tRNA selection. Rate constants for GTP hydrolysis (top) and peptide bond formation (bottom) were measured at $2.25 \mu \mathrm{M}$ ribosome complex and substoichiometric ternary complex in the absence or presence of $5 \mu \mathrm{M}$ paromomycin or $5 \mu \mathrm{M}$ streptomycin. Reactions were performed as described by Cochella and Green (2005a). (Reprinted, with permission, from Cochella et al. 2006 [Nature Publishing Group].) rating concentrations of ribosome complexes, it is likely that the observed decreased rates reflect deficiencies in steps distinct from binding of the ternary complex to the ribosome. These data provide strong evidence for an essential role for these nucleotides in the process of tRNA selection. These data corroborate conclusions drawn from biochemical studies that probed the importance of interactions in this region by disrupting contacts within the codon:anticodon helix itself (Fahlman et al. 2006; Gromadski et al. 2006).

We next looked at the effects of substitutions in the decoding region on the related reaction of peptide release. For these experiments, ribosomal initiation complexes were formed as above, but using an mRNA that substitutes for the Trp codon a stop codon (UAG) recognized by the class I release factor RF1. The resulting ribosome complexes were then reacted with varying concentrations of RF1 and the rates of peptide release were measured as described previously (Youngman et al. 2004). Although there are defects in peptide release associated with mutations in these positions, these defects are overcome at higher concentrations of RF1 (Fig. 2). These data indicate that the predominant effect of these mutations is on RF1 binding and that the identity of these nucleotides is less important for specifying the downstream catalytic events of peptide hydrolysis.

Taken together, these two sets of experiments indicate that the identity of the decoding center nucleotides is important for the two related processes of tRNA selection and release factor recognition. The discrepancies in how these nucleotide changes affect these reactions suggest, however, that they do not have the same role in the two processes. As discussed above, structural studies indicate that all three nucleotides undergo substantial conformational rearrangements on binding cognate tRNA during tRNA selection. The simplest interpretation of our data is that changes at these positions in the rRNA affect the stability of this flipped-out state of the decoding center, thus diminishing the induced fast rates of GTPase activation and accommodation. The data for the release reaction are more complicated and show that

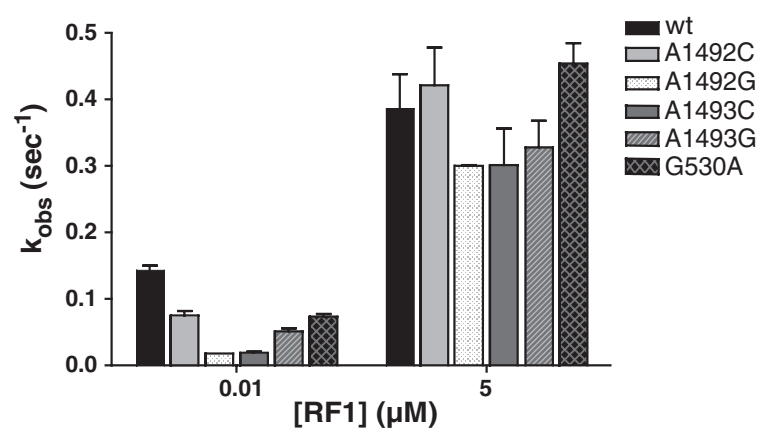

Figure 2. Decoding site mutants are defective for peptide release at low release factor concentrations. Rate constants for peptide release were measured on wild-type and mutant ribosomes at either $10 \mathrm{~nm}$ ribosome complex and $10 \mathrm{~nm} \mathrm{RF1}$ (left) or $250 \mathrm{~nm}$ ribosome complex and $5 \mu \mathrm{M}$ RF1 (right). Reactions were performed as described by Youngman et al. (2004), except that a high-fidelity polyamine-based buffer (Gromadski and Rodnina 2004a) was used. 
reasonably mild effects of the mutations on binding of RF1 to the ribosome are overcome with higher concentrations of release factor. These data indicate that the identity of these nucleotides is not essential for catalysis per se and that their mutation may simply impose a structure on the region that is unfavorable for release factor binding. For example, specific positive interactions between the nucleotides and the RF may be disrupted (or destabilized). Alternatively, the induced state of the region (flipped-out conformation) may not be favorable for peptide release, and the wild-type identity of these nucleotides may be optimized for stable docking within helix 44 in the absence of cognate RNA:RNA duplex. In this case, mutation might destabilize the flipped-in state of the decoding center and thus allow these nucleotides to compete directly with RF binding.

\section{EFFECTS OF AMINOGLYCOSIDE ANTIBIOTICS ON TRNA SELECTION AND PEPTIDE RELEASE}

The interactions of aminoglycoside antibiotics (such as paromomycin and streptomycin) with the ribosome decoding center are well characterized structurally and based on their effects on the process of tRNA selection. These compounds are generally known for their clear effects in stimulating miscoding on the ribosome during translation. We next looked at the effects of these antibiotics on the activity of ribosomes bearing mutations in the decoding center nucleotides discussed above. As previously reported, paromomycin has little effect on GTPase activation and accommodation in wild-type ribosomes, whereas streptomycin has a mild inhibitory effect on both reactions (Pape et al. 2000; Gromadski and Rodnina 2004b). These kinetic results have been rationalized from a structural perspective based on the observation that closure of the small ribosomal subunit is stabilized by both compounds but that the extent of closure is more significant with paromomycin than with streptomycin (Carter et al. 2000). Moreover, it has been shown that when both antibiotics are supplied in the same reaction, the biochemical read-out is one characteristic of streptomycin alone, as though this compound is dominant and prevents full closure of the subunit (Gromadski and Rodnina 2004b). Not unexpectedly, both paromomycin and streptomycin substantially relieve the defects associated with the decoding center mutations, nearly to wild-type levels in the case of the GTPase activation assay (see Fig. 1). These data are consistent with the idea that mutation of the decoding center nucleotides destabilizes interactions with the codon:anticodon helix and that these defects can be suppressed by artificially inducing closure of the subunit with these compounds. Other related studies have similarly shown that these antibiotics can suppress a variety of defects in the decoding center including mismatches in the codon:anticodon helix and the loss of critical 2'-OH groups in this same region (Pape et al. 2000; Fahlman et al. 2006).

We next asked. What are the effects of these same compounds on the peptide release reaction? In striking contrast to its effects on tRNA selection, paromomycin

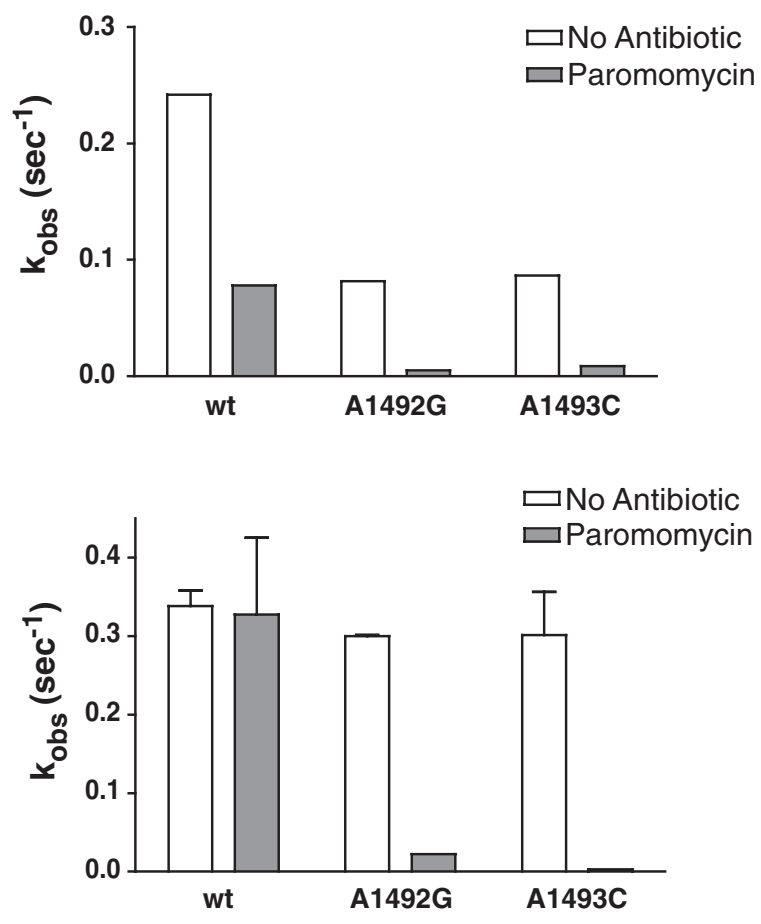

Figure 3. Paromomycin potentiates the defects of decoding site mutants in peptide release. Rate constants for peptide release were measured for wild-type and selected decoding site mutant ribosomes in the absence or presence of $50 \mu \mathrm{M}$ paromomycin. Reactions were performed at either $50 \mathrm{~nm}$ ribosome complex and $50 \mathrm{~nm}$ RF1 (top) or $250 \mathrm{~nm}$ ribosome complex and $5 \mu \mathrm{M}$ RF1 (bottom).

substantially inhibits RF1-catalyzed peptide release on wild-type ribosomes at low concentrations of RF1 (relative to its known $\mathrm{K}_{1 / 2}$ ), although this inhibition is overcome at higher concentrations of RF1 (Fig. 3). These data suggest that paromomycin affects the release reaction by simply inhibiting release factor binding. We next looked at the effects of paromomycin on the release defects associated with mutations in the decoding center nucleotides and found that paromomycin further antagonized the defects of these mutations, rather than suppressing them (Fig. 3). A model that reconciles these results is that stabilization of the flipped-out conformation of the decoding center is in some way unfavorable for the release reaction. In the case of wild-type ribosomes, the effects of paromomycin binding can be overcome by high concentrations of the release factor. We are currently determining whether the defects of the variant ribosomes can similarly be overcome with higher release factor concentrations.

\section{CONCLUSIONS}

The experiments reported in this study provide insight into the regulated function of the universally conserved decoding center of the ribosome. This RNA-rich site is engaged by distinct classes of molecules during translation - the tRNAs and the release factors - that must both recognize a codon in the mRNA with exceptionally high 
fidelity and in turn must activate related downstream catalytic events in the large ribosomal subunit. In the absence of specific knowledge, it had seemed reasonable that these two processes would be highly related, albeit one RNAbased and the other protein-based, and thus would depend on similar molecular components for optimal function. What these studies have revealed is that tRNA selection and stop codon recognition during peptide release are in fact orchestrated by quite distinct mechanisms in the decoding center. Universally conserved nucleotides in the decoding center, although important at some level for both processes, are more specifically important for tRNA selection where they engage the minor groove of the codon:anticodon helix to activate efficient downstream GTPase activation and accommodation. In the release reaction, defects associated with these nucleotide substitutions are easily overcome at increasing concentrations of release factor with no residual effects on catalysis. Similarly, paromomycin has disparate effects on tRNA selection and peptide release, being overall stimulatory in the former case and overall inhibitory in the latter. In light of the well-documented effects of paromomycin on ribosome structure, we argue that tRNAs and release factors recognize distinct states of the ribosome that minimally differ in the position of A1492/A1493. Although tRNA selection depends on the flipped-out conformation of these adenosines (the "on" configuration), release factors apparently bind to the flipped-in or "off" state of the decoding center.

A number of different lines of evidence would suggest that tRNA selection and peptide release function, as they now exist, arose at different points during the evolution of the translation apparatus. Most striking among these arguments is the observation that bacterial and eukaryotic release factors are not related at the sequence level and thus apparently arose as independent solutions to the same problem (Frolova et al. 1999). In light of this, perhaps the observation that tRNA selection and peptide release depend on such distinct molecular mechanisms is less surprising. The creation of an active site that must engage a variety of distinct molecular partners is a formidable challenge, and these studies only begin to decipher how evolution has solved this problem in the protein synthesis apparatus.

\section{ACKNOWLEDGMENTS}

We thank V. Ramakrishnan and M. Rodnina for helpful discussions, the National Institutes of Health for funding, and the Howard Hughes Medical Institute for salary support.

\section{REFERENCES}

Bouadloun F., Donner D., and Kurland C.G. 1983. Codonspecific missense errors in vivo. EMBO J. 2: 1351.

Brunelle J.L., Youngman E.M., Sharma D., and Green R. 2006. The interaction between C75 of tRNA and the A loop of the ribosome stimulates peptidyl transferase activity. RNA 12: 33.

Carter A.P., Clemons W.M., Brodersen D.E., Morgan-Warren R.J., Wimberly B.T., and Ramakrishnan V. 2000. Functional insights from the structure of the 30S ribosomal subunit and its interactions with antibiotics. Nature 407: 340.

Caskey C.T., Beaudet A.L., Scolnick E.M., and Rosman M. 1971. Hydrolysis of fMet-tRNA by peptidyl transferase. Proc. Natl. Acad. Sci. 68: 3163.

Cochella L. and Green R. 2005a. An active role for tRNA in decoding beyond codon:anticodon pairing. Science 308: 1178 .

2005b. Fidelity in protein synthesis. Curr. Biol. 15: R536.

Cochella L., Brunelle J.L., and Green R. 2006. Mutational analysis reveals two independent molecular requirements during transfer RNA selection on the ribosome. Nat. Struct. Mol. Biol.

Fahlman R.P., Olejniczak M., and Uhlenbeck O.C. 2006. Quantitative analysis of deoxynucleotide substitutions in the codon-anticodon helix. J. Mol. Biol. 355: 887.

Freistroffer D.V., Kwiatkowski M., Buckingham R.H., and Ehrenberg M. 2000. The accuracy of codon recognition by polypeptide release factors. Proc. Natl. Acad. Sci. 97: 2046.

Frolova L.Y., Tsivkovskii R.Y., Sivolobova G.F., Oparina N.Y., Serpinsky O.I., Blinov V.M., Tatkov S.I., and Kisselev L.L. 1999. Mutations in the highly conserved GGQ motif of class 1 polypeptide release factors abolish ability of human eRF1 to trigger peptidyl-tRNA hydrolysis. RNA 5: 1014.

Gromadski K.B. and Rodnina M.V. 2004a. Kinetic determinants of high-fidelity tRNA discrimination on the ribosome. Mol. Cell 13: 191.

2004b. Streptomycin interferes with conformational coupling between codon recognition and GTPase activation on the ribosome. Nat. Struct. Mol. Biol. 11: 316.

Gromadski K.B., Daviter T., and Rodnina M.V. 2006. A uniform response to mismatches in codon-anticodon complexes ensures ribosomal fidelity. Mol. Cell 21: 369.

Jorgensen F., Adamski F.M., Tate W.P., and Kurland C.G. 1993. Release factor-dependent false stops are infrequent in Escherichia coli. J. Mol. Biol. 230: 41.

Ogle J.M. and Ramakrishnan V. 2005. Structural insights into translational fidelity. Annu. Rev. Biochem. 74: 129.

Ogle J.M., Murphy F.V., Tarry M.J., and Ramakrishnan V. 2002. Selection of tRNA by the ribosome requires a transition from an open to a closed form. Cell 111: 721.

Ogle J.M., Brodersen D.E., Clemons W.M., Jr., Tarry M.J., Carter A.P., and Ramakrishnan V. 2001. Recognition of cognate transfer RNA by the $30 \mathrm{~S}$ ribosomal subunit. Science 292: 897.

Pape T., Wintermeyer W., and Rodina M.W. 1999. Induced fit in initial selection and proofreading of aminoacyl-tRNA on the ribosome. Eur. J. Mol. Biol. 18: 3800 .

2000. Conformational switch in the decoding region of $16 \mathrm{~S}$ rRNA during aminoacyl-tRNA selection on the ribosome. Nat. Struct. Biol. 7: 104.

Parker J. and Holtz G. 1984. Control of basal-level codon misreading in Escherichia coli. Biochem. Biophys. Res. Commun. 121: 487.

Petry S., Brodersen D.E., Murphy F.V., Dunham C.M., Selmer M., Tarry M.J., Kelley A.C., and Ramakrishnan V. 2005. Crystal structures of the ribosome in complex with release factors RF1 and RF2 bound to a cognate stop codon. Cell 123: 1255.

Powers T. and Noller H.F. 1990. Dominant lethal mutations in a conserved loop in 16S rRNA. Proc. Natl. Acad. Sci. 87: 1042.

Schmeing T.M., Huang K.S., Strobel S.A., and Steitz T.A. 2005. An induced-fit mechanism to promote peptide bond formation and exclude hydrolysis of peptidyl-tRNA. Nature 438: 520.

Youngman E.M. and Green R. 2005. Affinity purification of in vivo-assembled ribosomes for in vitro biochemical analysis. Methods 36: 305.

Youngman E.M., Brunelle J.L., Kochaniak A.B., and Green R. 2004. The active site of the ribosome is composed of two layers of conserved nucleotides with distinct roles in peptide bond formation and peptide release. Cell 117: 589. 


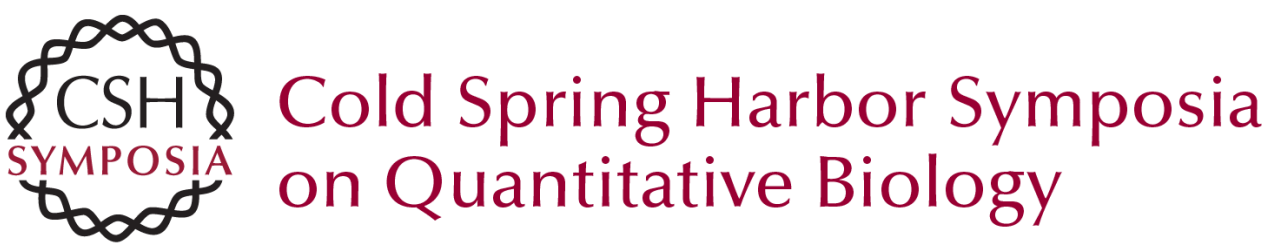

\title{
Two Distinct Conformations of the Conserved RNA-rich Decoding Center of the Small Ribosomal Subunit Are Recognized by tRNAs and Release Factors
}

\author{
E.M. YOUNGMAN, L. COCHELLA, J.L. BRUNELLE, et al.
}

Cold Spring Harb Symp Quant Biol 2006 71: 545-549

Access the most recent version at doi:10.1101/sqb.2006.71.036

References This article cites 24 articles, 7 of which can be accessed free at: http://symposium.cshlp.org/content/71/545.full.html\#ref-list-1

\section{License}

Email Alerting Receive free email alerts when new articles cite this article - sign up in Service the box at the top right corner of the article or click here. 